\title{
AOR
}

Selected Papers of \#AoIR2021: The 22nd Annual Conference of the Association of Internet Researchers

Virtual Event / 13-16 Oct 2021

\section{THE CHESHIRE CAT OF SOCIAL MEDIA: VALUES IN PLATFORM POLICIES}

\author{
Rebecca Scharlach \\ The Hebrew University of Jerusalem \\ Blake Hallinan \\ The Hebrew University of Jerusalem \\ Limor Shifman \\ The Hebrew University of Jerusalem
}

Value is fundamental for social media platforms, not only in the economic sense but also in the sense of normative principles like community and free speech. Policy documents are pivotal sites for the expression of values and present a public-facing account of the roles and responsibilities assigned to various actors, including individual users, third parties, governments and social media corporations. While prior research has examined the construction of individual values such as privacy and transparency in platform policies, there has been no holistic account of the values invoked in these documents. Combining a dictionary-based analysis with a qualitative content analysis, we present the first comprehensive study of the values presented in the policy documents of five major social media platforms: Facebook, Instagram, YouTube, Twitter, and TikTok.

Our analysis reveals that policy documents invoke a large number of values that seemingly point to conflicting priorities and commitments. However, these values can be organized in four overarching principles that were consistent across platforms:

community, expression, privacy, and improvement. Each principle assigns responsibility for the enactment of these values and by that allows platforms to limit their ethical responsibility for executing the values they publicly promote. Values can thus be described as the 'Cheshire cat' of social media platform policies - they look magical, but once touched, may assume a different shape, pop up in an alternative location, or even disappear. 